\title{
Battling hypertension
}

$\mathbf{E}$ veryone has heard the mantra of healthy living: eat right, exercise and get plenty of rest. Unfortunately, few people actually live that way, and many are paying for it. For example, in the United States alone, about one out of every six people suffers from high blood pressure. It is ironic that modern society, with its emphasis on high achievement and a fast-paced lifestyle, has fostered the conditions for hypertension to run rampant but has also made it possible to investigate and treat this condition effectively.

Doctors do not fully understand the primary causes of hypertension, but it is clear that stress, inactivity and an unhealthy diet contribute. Obviously, heredity also plays a large part. The good news is physicians can use a wide variety of drugs to safely lower blood pressure in almost anyone. Moreover, researchers are working to understand both genetic predisposition toward hypertension and the specific molecular mechanisms underlying it, as the study on page 554 of this issue illustrates.

Approximately one-third of African-Americans and a quarter of Caucasian-Americans have high blood

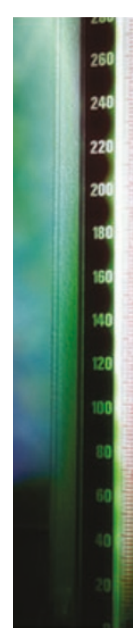
pressure. In addition, older people are more susceptible, as are obese individuals. Hypertension is often called the 'silent killer' because it causes no symptoms in most people in the early stages and is usually detected only through routine doctor visits. As a result, it is estimated that only two-thirds of people affected have been diagnosed and are undergoing treatment.

Blood pressure in the arteries is defined by two values: the systolic pressure (when the heart beats) and the diastolic pressure (when the heart rests between beats). It is written as systolic/diastolic and is measured in millimeters of mercury. Normal pressure is $120 / 80$, and pressures of 140/90 or above are classified as hypertension. If pressures above that level are sustained for a long period, the heart may become enlarged, organ damage can result, and heart attacks and strokes are much more likely.

The body can control blood pressure at many levels. For example, to decrease the pressure in the arteries, the heart can pump less rapidly, the kidneys can reduce the amount of fluid in the bloodstream, or blood vessels can widen. These mechanisms are controlled primarily by two systems: the sympathetic division of the autonomous nervous system and the kidneys.

The kidneys control blood pressure through the renin-angiotensinaldosterone system, the subject of the paper on page 554. To increase blood pressure, the kidneys secrete the enzyme renin, which cleaves angiotensin to create angiotensin I, which is further processed by angiotensinconverting enzyme (ACE) to yield angiotensin II. This polypeptide triggers the constriction of arteriole walls, and it also causes the adrenal glands to release aldosterone. Aldosterone promotes the retention of sodium, and hence the retention of water, which increases blood volume and thus blood pressure.

The sympathetic division controls the fight-orflight response by stimulating the adrenal glands to secrete adrenalin and norepineprhine. The heart beats faster, and within the skeletal muscles and other systems that are critical for quick action, blood vessels dilate. The kidneys are also stimulated to retain sodium and water.

Moderate exercise and eating a healthy, low-salt diet can reduce blood pressure adequately in some people. However, if lifestyle changes alone do not help, there is an enormous variety of antihypertensive drugs to try. Each class acts at a different step, and only a few are listed here. Diuretics help the kidneys eliminate salt and water. Beta-blockers limit the effect of the sympathetic nervous system. ACE inhibitors prevent the production of angiotensin II. Angiotensin-receptor blockers specifically prevent the interaction between angiotensin II and its seven-transmembrane receptor. And antagonists dampen the effects of the aldosterone receptor. Many of these drugs may be used in combination to tailor treatment to a patient's individual response.

Antihypertensive drugs have become big business for the pharmaceutical industry. For those whose genetic makeup is refractive to control by diet and exercise, there is no choice but to rely on medical management of their high blood pressure. For others, it is far easier to take a pill than to make significant lifestyle changes-a distressing commentary on the priorities of our contemporary culture. But at least that option exists. 